\title{
Platelet-rich plasma inhibits RANKL-induced osteoclast differentiation through activation of Wnt pathway during bone remodeling
}

\author{
DONGYUE WANG*, YAJUAN WENG* , SHUYU GUO*, YUXIN ZHANG, TINGTING ZHOU, \\ MENGNAN ZHANG, LIN WANG and JUNQING MA
}

Jiangsu Key Laboratory of Oral Diseases, Nanjing Medical University, Nanjing, Jiangsu 210029, P.R. China

Received February 7, 2017; Accepted November 2, 2017

DOI: $10.3892 /$ ijmm.2017.3258

\begin{abstract}
Platelet-rich plasma (PRP) is used in the clinic as an autologous blood product to stimulate bone regeneration and chondrogenesis. Numerous studies have demonstrated that PRP affects bone remodeling by accelerating osteoblast formation. With the research perspective focusing on osteoclasts, the present study established a mouse model of mandibular advancement to examine the effect of PRP on osteoclast differentiation induced by modification of the dynamics of the temporomandibular joint (TMJ). The lower incisors of the mice were trimmed by $1 \mathrm{~mm}$ and the resultant change in mandibular position during the process of eating induced condylar adaptation to this change. PRP significantly increased the bone mass and decreased osteoclastic activity, in vitro as well as in vivo. Mechanistically, the reduced expression of receptor activator of nuclear factor- $\kappa \mathrm{B}$ ligand (RANKL)-induced differentiation marker genes, including nuclear factor of activated T-cells, cytoplasmic 1 , c-fos and tartrate-resistant acid phosphatase, and that of the resorptive activity marker genes such as cathepsin $\mathrm{k}$, carbonic anhydrase 2 and matrix metalloproteinase 9, indicated that PRP suppresses RANKL-induced osteoclast differentiation. A microarray analysis revealed that several genes associated with the Wnt pathway were differentially expressed, which indicated the involvement of this pathway in osteoclast differentiation. Furthermore, the activation of the Wnt pathway was verified by reverse transcription-quantitative polymerase chain reaction and immunoblot analysis of Dickkopf-related
\end{abstract}

Correspondence to: Professor Junqing Ma, Jiangsu Key Laboratory of Oral Diseases, Nanjing Medical University, 136 Hanzhong Road, Nanjing, Jiangsu 210029, P.R. China

E-mail: jma@njmu.edu.cn

"Contributed equally

Key words: platelet-rich plasma, temporomandibular joint modification, bone formation, Dickkopf-related protein $1, \beta$-catenin protein 1 and $\beta$-catenin. The results of the present study indicated that PRP inhibits osteoclast differentiation through activation of the Wnt pathway.

\section{Introduction}

Angle class II malocclusion is a common condition that presents most commonly as mandibular retrusion (1). Treatment of this condition involves the use of functional appliances to stimulate mandibular growth by forward posturing of the mandible. During this process, a series of morphological and histological changes are observed in the region of the temporomandibular joint (TMJ), which manifest as reconstruction of the condyle and glenoid fossa (2). Studies have demonstrated that mandibular advancement may induce endochondral bone formation in the condyle (3-5). The present study established a mouse model of stepwise mandibular advancement to study bone homeostasis associated with TMJ modification in the absence and presence of platelet-rich plasma (PRP).

The coordination between bone matrix formation by osteoblasts and bone resorption by osteoclasts maintains bone homeostasis (6). Shifting of the balance either towards boneresorbing osteoclasts or bone-forming osteoblasts is implicated in the causation of several bone disorders. Osteoclasts are multinucleated cells of hematopoietic origin responsible for bone resorption and have a critical role in maintaining bone remodeling and mineral homeostasis $(7,8)$. Two cytokines, the macrophage colony-stimulating factor (M-CSF) and the receptor activator of nuclear factor- $\kappa \mathrm{B}$ ligand (RANKL) are known to regulate osteoclast differentiation (9).

PRP refers to plasma with an enhanced concentration of platelets. As a natural source of a variety of growth factors, PRP has an important role in bone repair, cell proliferation and differentiation during tissue regeneration (10). PRP has been demonstrated to stimulate bone regeneration and healing by inducing proliferation and differentiation of osteoblasts $(11,12)$. However, its effect on RANKL-induced osteoclast differentiation has remained to be fully elucidated.

The aim of the present study was to evaluate the effect of PRP on osteoclast differentiation during mandibular condyle remodeling, and to determine the underlying molecular mechanisms involved in this biological process. 


\section{Materials and methods}

Animals and experimental design. A total of 100, 3 week-old femaleBALB/cmiceweremaintainedinatemperature-controlled room $\left(24 \pm 1^{\circ} \mathrm{C}\right)$ with relative humidity $(50 \pm 10 \%)$ at the animal center of Nanjing Medical University (Nanjing, China) with free access to mouse chow and water and underwent a $12 \mathrm{~h}$ light/dark cycle. All animal experimentation was performed according to protocols approved by the Experimental Animal Care and Use Committee of Nanjing Medical University (Nanjing, China). Mice were provided by the Model Animal Research Center of Nanjing University (Nanjing, China).

Induction of mandibular forward movement was performed as described previously (13). In brief, in 4-week-old mice, both sides of the lower incisors were trimmed by $1 \mathrm{~mm}$ at the incisal third every 3 days until they were sacrificed. This was performed to induce a mandibular protrusion movement that occurs when mice are feeding.

Four-week-old female mice were divided into two groups ( $n=5$ in each): The negative control group (received normal saline) and the PRP group (received PRP treatment). Normal saline or PRP were directly injected into the articular cavity of the TMJ by a micro syringe once a week. After injection, the animals were allowed to recover and were sacrificed at 7,14 and 28 days after mandibular forward movement.

Preparation of PRP. Whole blood collected from age-matched female BALB/c mice was transferred to sterile tubes and mixed with sodium citrate (3.8\%) at a ratio of 9:1 to prevent coagulation. PRP was enriched by a two-step centrifugation method as described previously (14). The tubes were centrifuged at $600 \mathrm{x}$ g for $10 \mathrm{~min}$ at room temperature and the whole blood was separated into three phases: platelet-poor plasma (top), buffy coat (middle) and erythrocytes (bottom). The top and middle liquid phases were transferred to a fresh tube and centrifuged again at 1,200 $\mathrm{x} g$ for $15 \mathrm{~min}$. The upper half of the supernatant plasma was discarded and the lower half was blended thoroughly to yield PRP. PRP was activated with $10 \%$ calcium chloride solution and 5,000 units of bovine thrombin to induce the formation of a gel, which was then stored at $-20^{\circ} \mathrm{C}$.

Micro-computed tomography (micro-CT) analysis. To assess differences in the density of subchondral bone and the degree of bone resorption, the condylar bone was cleared of marrow and soft tissue and then fixed overnight in 70\% ethanol (15). The specimens were then scanned at a slice thickness of $18 \mu \mathrm{m}$ using a Skyscan 1176 micro-CT device (Skyscan, Kontich, Belgium) at $50 \mathrm{kV}$ and $456 \mu \mathrm{A}$. Images were reconstructed and analyzed using NRecon version 1.6 and CTAn version 1.31.8.1 software (Bruker, Billerica, MA, USA). The area of condylar cartilage and subchondral bone in the sample was defined as the region of interest (ROI). A total of five consecutive images from the ROI were used for three-dimensional reconstruction and analysis.

The parameters analyzed included the trabecular bone volume per total volume (BV/TV), trabecular number (Tb.N), trabecular separation (Tb.Sp) and trabecular thickness (Tb.Th). The operator who performed the scanning analysis was blinded to the group identity of each sample.
Histological analysis. Mandibular condyles from the control and experimental animals were resected and fixed in $10 \%$ buffered formalin for $24 \mathrm{~h}$, decalcified in 10\% EDTA (pH 7.4) for 21 days, embedded in paraffin and cut into $4-\mu \mathrm{m}$ thick sections. The sections were stained for total collagen, as well as with tartrate-resistant acid phosphatase (TRAP) and Goldner's trichrome as described previously $(16,17)$. The histomorphometric parameters determined included the percentage of collagen area, the number of osteoclasts per bone perimeter $(\mathrm{OcN} / \mathrm{BP})$ and the number of osteoblasts per bone perimeter (ObN/BP) (18). Two examiners blinded to the group identity analyzed the slides.

Mouse osteoclast culture. Bone marrow cells and hematopoietic cells were extracted from the tibias and femurs of three-week-old BALB/c female mice under general anesthesia, as described previously (19). Cells were cultured in $\alpha$-minimum essential medium ( $\alpha$-MEM) with $15 \%$ fetal bovine serum (FBS) (both from Gibco; Thermo Fisher Scientific, Inc., Waltham, MA, USA) and $1 \%$ penicillin-streptomycin (PS) at $37^{\circ} \mathrm{C}$ and $5 \% \mathrm{CO}_{2}$ for $3 \mathrm{~h}$ to remove adherent cells. Non-adherent cells were seeded onto new plates and cultured in $\alpha$-MEM with M-CSF (20 ng/ml; Sigma-Aldrich; Merck KGaA, Darmstadt, Germany) for 3 days, which resulted in the growth of bone marrow-derived macrophages (BMMs).

For osteoclast differentiation and TRAP activity staining, BMMs were seeded in 6-well plates and cultured in the presence of M-CSF (20 ng/ml) and RANKL (20 ng/ml; Sigma-Aldrich; Merck KGaA) with or without $1 \%$ PRP for 3 days.

TRAP staining. The protocol used for cell TRAP staining was in accordance with that of a previous study (20). In brief, cells were fixed in $4 \%$ paraformaldehyde and stained for TRAP activity with $0.1 \mathrm{M}$ acetate solution ( $\mathrm{pH}$ 5.0) containing $6.76 \mathrm{mM}$ sodium tartrate, $0.1 \mathrm{mg} / \mathrm{ml}$ naphthol AS-MX phosphate and $0.5 \mathrm{mg} / \mathrm{ml}$ Fast Red Violet at $37^{\circ} \mathrm{C}$ for $30 \mathrm{~min}$. Multinucleated cells expressing TRAP and with $>3$ nuclei were identified as osteoclasts.

Alizarin Red $S$ (ARS) staining. Bone marrow stromal cells (BMSCs) flushed from the femurs and tibias were cultured in $\alpha$-MEM containing $10 \% \mathrm{FBS}$ and $1 \% \mathrm{PS}$ at $37^{\circ} \mathrm{C}$ and $5 \% \mathrm{CO}_{2}$ for 3 days, followed by removal of non-adherent cells by replacing the medium. For osteogenic differentiation, BMSCs were seeded at a density of $1 \times 10^{5}$ cells/well in 12 -well plates in a mineralized solution containing $\alpha$-MEM, $10 \%$ FBS, $10 \mathrm{mM} \beta$-glycerolphosphate, $10 \mathrm{nM}$ dexamethasone and $50 \mathrm{mg} / \mathrm{ml}$ ascorbate phosphate, with and without addition $1 \%$ PRP. After 12 days of ossification induction, mineral deposition was assessed by ARS staining. A microplate reader was used to capture micrographs of mineralized nodules. ARS staining was performed as per the manufacturer's instructions (Beyotime Institute of Biotechnology, Haimen, China).

Microarray and gene expression analysis. Total RNA was extracted from condyle and glenoid fossa samples of test and control mice using TRIzol reagent (Invitrogen; Thermo Fisher Scientific, Inc.) at 14 days. A NanoDrop ND-1000 was used for RNA quantification and quality assessment, while RNA 
A
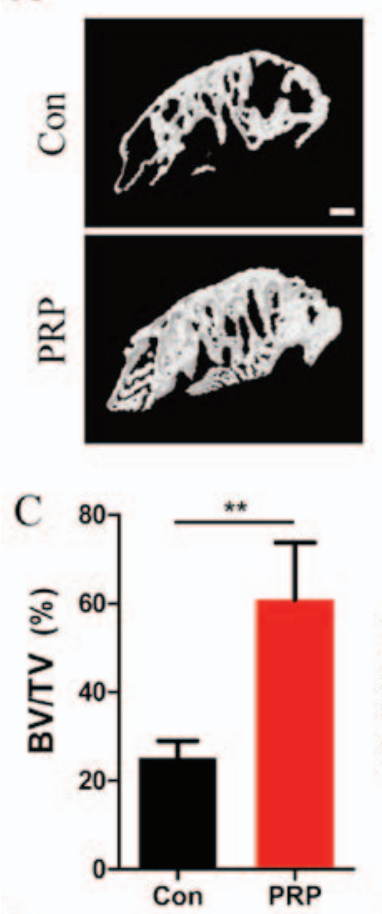

D

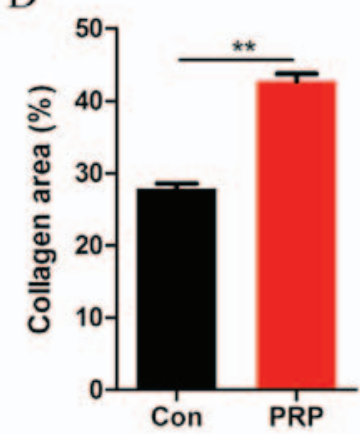

B

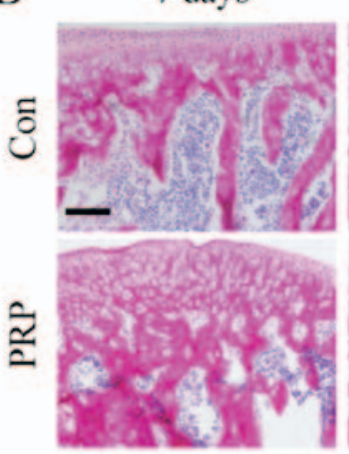

14 days

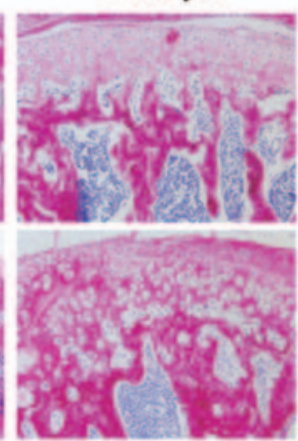

28 days

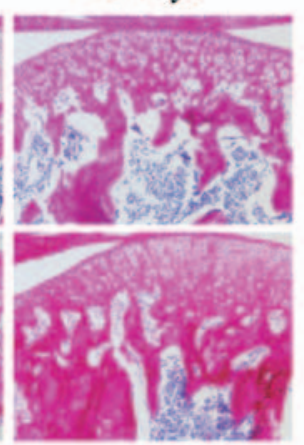

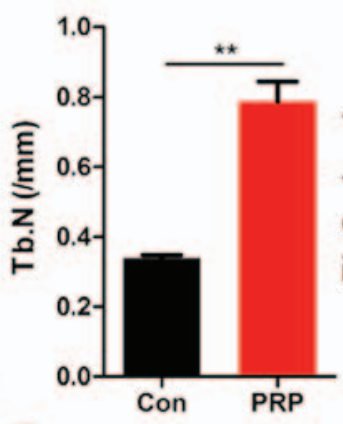

E

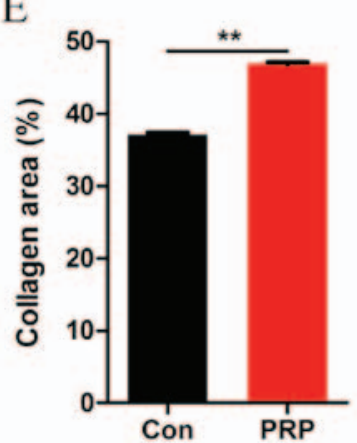

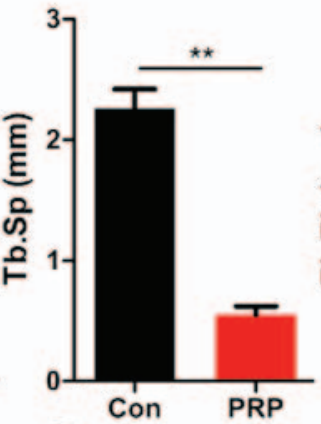

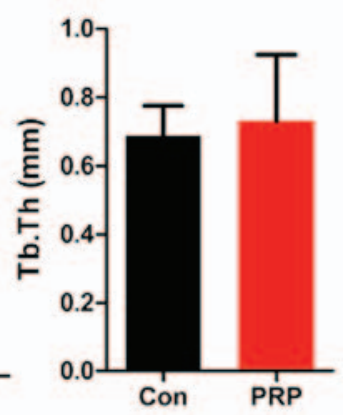

$\mathrm{F}$

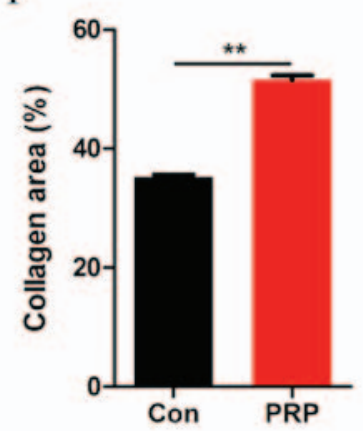

Figure 1. PRP alleviates bone loss in a mouse model of mandibular advancement. (A) Four-week-old mice were sacrificed on day 28 after the first mandibular forward movement, and radiographs of the transverse sections of the mandibular condyles were obtained using a micro-CT scanner. Scale bar, $100 \mu \mathrm{m}$. (B) Representative photomicrographs of sections subjected to histochemical staining for total collagen. Scale bar, $100 \mu \mathrm{m}$. (C) BV/TV, Tb.N and Tb.Sp of the mandibular condyles were determined by analysis of micro-CT data with Xelis software. No significant difference was observed with respect to trabecular thickness between the control and experimental groups $(\mathrm{P}>0.05)$. (D-F) The proportion of red-stained collagenous area within the total area in the entire section, on (D) day 7, (E) day 14 and (F) day 28 in the PRP group was significantly greater than that in the control group. Values are expressed as the mean \pm standard deviation $(n=5) .{ }^{* *} \mathrm{P}<0.01$. PRP, platelet-rich plasma; RANKL, receptor activator of nuclear factor- $\kappa \mathrm{B}$ ligand; $\mathrm{BV} / \mathrm{TV}$, bone volume per total volume; Tb.Sp, trabecular separation; Tb.Th, trabecular thickness; CT, computed tomography; Con, control.

integrity was assessed using standard denaturing agarose gel electrophoresis. The Whole Mouse Genome Oligo Microarray (4x44K; Agilent Technologies, Santa Clara, CA, USA) was used to investigate the transcriptional profiles of the samples; the array represented $>41,000$ transcripts.

RNA labeling and array hybridization were performed according to the Agilent One-Color Microarray-Based Gene Expression Analysis protocol (Agilent Technologies). Data extracted using Agilent Feature Extraction software (version 11.0.1.1) were normalized and analyzed using the GeneSpring GX v12.1 software package (Agilent Technologies). Differentially expressed genes were identified by fold-change screening.

Gene ontology (GO) analysis and Kyoto Encyclopedia of Genes and Genomes (KEGG) pathway enrichment analysis was performed to systematically identify the terms of differentially expressed genes and to identify the pathways associated with osteoclastic differentiation. The microarray data were deposited in the NCBI Gene Expression Omnibus (www.ncbi.nlm.nih.gov/geo/; accession no. GSE67644) (21). The procedure was performed by KangChen Bio-Tech (Shanghai, China). $\mathrm{P}<0.005$ was used as a cutoff threshold to identify significantly enriched GO terms and pathways.

Reverse transcription-quantitative polymerase chain reaction (RT-qPCR) for RNA analysis. Total RNA was isolated from osteoclasts which were treated with or without $1 \%$ PRP for 3 days using an RNA extraction kit (Takara Bio, Inc., Otsu, Japan) according to the manufacturer's protocol. The RNA was converted to complementary DNA using a Takara PrimeScript RT reagent kit (Takara Bio, Inc.). The gene expression level was assessed by qPCR using the ABI-7300 Real-Time PCR system (Applied Biosystems; Thermo Fisher Scientific, Inc.). The qPCR mixture contained $2 \mu \mathrm{l}$ of cDNA, $0.5 \mu \mathrm{l}$ of each 
A

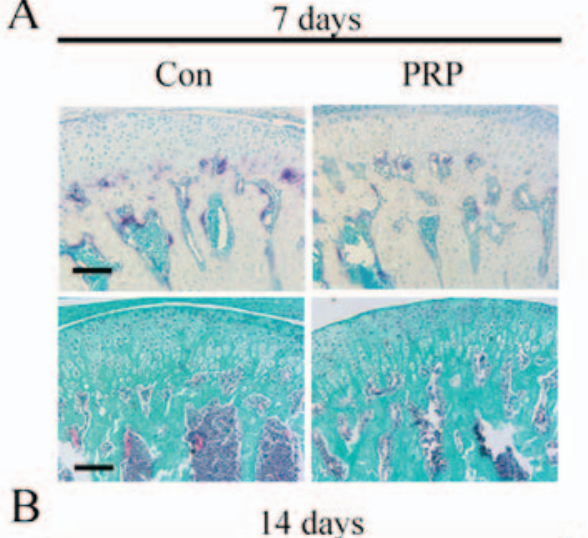

Con

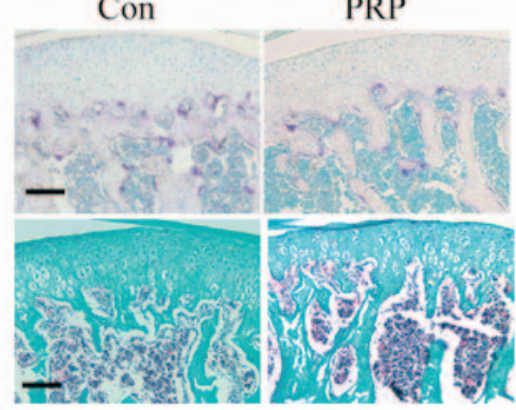

$\mathrm{C}$

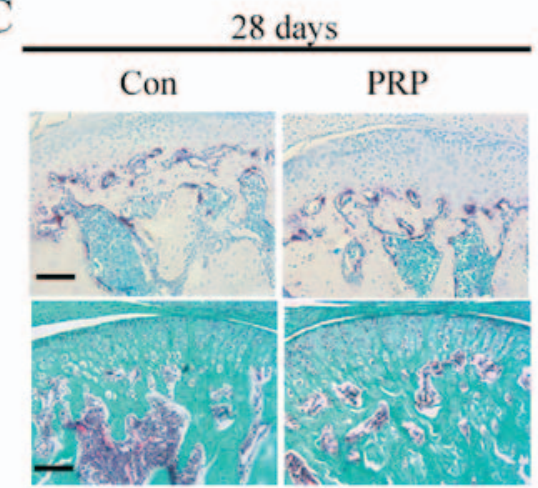

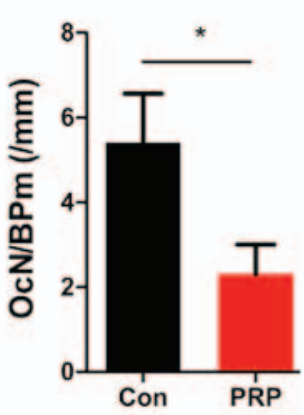
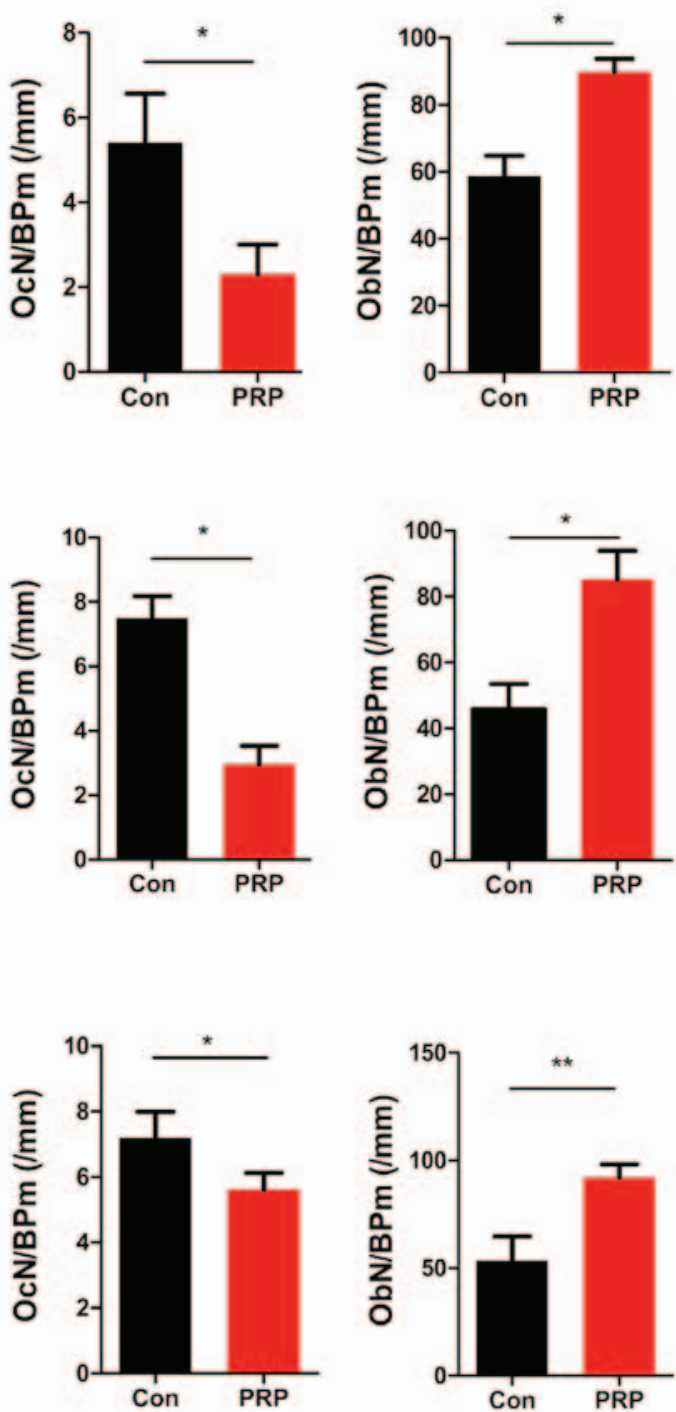

Figure 2. Effects of PRP on bone homeostasis and osteoclastic differentiation. The dissected mandibular condyles of day 7 (A), day 14 (B) and day 28 (C) were fixed, decalcified, embedded and sectioned (eft). The sections were stained for TRAP (top) and subjected to Goldner's trichrome staining (bottom). Scale bar, $100 \mu \mathrm{m}$. The OcN/BP and ObN/BP were determined by histomorphometric analysis (right). Values are expressed as the mean \pm standard deviation ( $\mathrm{n}=5$ ). $\mathrm{P}<0.05$ and ${ }^{* *} \mathrm{P}<0.01$. TRAP, tartrate resistant acid phosphatase; OcN, number of osteoclasts; ObN, number of osteoblasts; BP, bone perimeter; PRP, platelet-rich plasma; Con, control.

primer and $10 \mu \mathrm{l}$ of SYBR-Green in a final volume of $20 \mu \mathrm{l}$ $\left(95^{\circ} \mathrm{C}\right.$ for $30 \mathrm{sec} ; 40$ cycles of $95^{\circ} \mathrm{C}$ for $5 \mathrm{sec}, 60^{\circ} \mathrm{C}$ for $31 \mathrm{sec}$, $95^{\circ} \mathrm{C}$ for $15 \mathrm{sec}, 60^{\circ} \mathrm{C}$ for $1 \mathrm{~min}$ and $95^{\circ} \mathrm{C}$ for $15 \mathrm{sec}$ ).

The following primers were used: Glyceraldehyde 3-phosphate dehydrogenase (GAPDH) forward, 5'-ACCACAGTCCA TGCCATCAC-3' and reverse, 5'-TCCACCACCCTGTTGCT GTA-3'; nuclear factor of activated T-cells, cytoplasmic 1 (NFATc1) forward, 5'-CGAGTTCACATCCCACAG-3' and reverse, 5'-GACAGCACCATCTTCTTCC-3'; c-fos forward, 5'-CACTCTGGTCTCCTCCGT-3' and reverse, 5'-ATTCTCC GTTTCTCTTCCTC-3'; TRAP forward, 5'-CAGCAGCCAAG GAGGACTAC-3' and reverse, 5'-ACATAGCCCACACCGTT CTC-3'; cathepsin k (Ctsk) forward, 5'-CCCATCTCTGTG TCCATC-3' and reverse, 5'-AGTGCTTGCTTCCCTTCT-3'; carbonic anhydrase 2 (CAR2) forward, 5'-ATCCTTGCTCCC TTCTTC-3' and reverse, 5'-ATCCAGGTCACACATTCC-3'; matrix metalloproteinase 9 (MMP9) forward, 5'-TCACTTTC
CCTTCACCTTC-3' and reverse, 5'-ATTTGCCGTCCTTAT CGT-3'; Dickkopf-related protein 1 (Dkk1) forward, 5'-ATT CCAGCGCTGTTACTGTG-3' and reverse, 5'-GAATTGCTG GTTTGATGGTG-3'; $\beta$-catenin forward, 5'-TTCCTGAGC TGACCAAACTG-3' and reverse, 5'-GCACTATGGCAGAC ACCATC-3'; cyclin D1 forward, 5'-CGGATGAGAACA AGCAGA-3' and reverse, 5'-CGGTAGCAGGAGAGGAAG-3'. The $2^{-\Delta \Delta C q}$ method was used for quantification (22).

Western blot analysis. Western blot analysis was performed according to a previously described method (23). After osteoclasts were treated with or without $1 \%$ PRP for 3 days and 5 days, total cellular protein was extracted using a TPER Protein Extraction reagent kit (Thermo Fisher Scientific, Inc.) according to manufacturer's instructions. The protein concentrations were determined using a bicinchoninic acid protein assay kit (Thermo Fisher Scientific, Inc.). Equal amounts of 
protein $(50 \mu \mathrm{g})$ were separated by $10 \%$ sodium dodecyl sulfatepolyacrylamide gel electrophoresis (SDS-PAGE), transferred by electro-blotting onto a polyvinylidene difluoride membrane (EMD Millipore Corp., Billerica, MA, USA) and the membrane was incubated overnight at $4^{\circ} \mathrm{C}$ with the following primary antibodies: Mouse Dkk1 antibody (1:1,000 dilution; cat. no. AF1765; R\&D Systems, Minneapolis, MN, USA), rabbit $\beta$-catenin antibody (1:1,000 dilution; cat. no. 8480; Cell Signaling Technology, Boston, MA, USA), rabbit anti-GAPDH (1:5,000 dilution; cat. no. AP0063; Bioworld, Irving, TX, USA), followed by incubation with horseradish peroxidaseconjugated secondary antibodies (cat. no. ZB-2301; Origene Technologies, Beijing, China) at room temperature for $1 \mathrm{~h}$. The protein bands were visualized with ECL substrate solution (GE Healthcare Life Sciences, Little Chalfont, UK) for $1 \mathrm{~min}$, and images were captured using a MicroChemiluminescence system 4.2 (DNR, Jerusalem, Israel). Semi-quantitative analyses were performed using ImageJ v.1.45 software (National Institutes of Health, Bethesda, NJ, USA).

Statistical analysis. Values are expressed as the mean \pm standard deviation. Experiments were conducted separately at least 3 times. SPSS 19.0 (IBM Corp., Armonk, NY, USA was used for statistical analysis. Student's t-test was used to assess inter-group differences. $\mathrm{P}<0.05$ was considered indicative of a statistically significant difference.

\section{Results}

Injection of PRP is associated with bone reconstruction. To investigate the effect of PRP on bone mass, micro-CT was used to characterize the bone phenotype and to quantitatively analyze the relevant parameters of condylar subchondral bone (Fig. 1). Micro-CT analysis of the mandibular condyle from 4-week-old mice indicated significant differences between the two groups (Fig. 1A). Mice in the PRP group exhibited a higher BV/TV $(\mathrm{P}<0.01)$, and increased Tb.N $(\mathrm{P}<0.01)$ and a reduced Tb.Sp $(\mathrm{P}<0.01)$. However, no significant difference in Tb.Th was observed between the two groups (Fig. 1C). Together, these results suggested a significant effect of PRP on bone formation in vivo under this specific physiological conditions of bone remodeling. This was also supported by the results of total collagen staining of TMJ sections at 7, 14 and 28 days (Fig. $1 \mathrm{~B}$ and $\mathrm{D}-\mathrm{F}$ ).

PRP facilitates osteoblast differentiation but inhibits osteoclast formation in condyle subchondral bone. To examine the effects of PRP on bone homeostasis and osteoclastic differentiation, differences in histological staining were compared between the experimental and control samples. TRAP staining of TMJ sections from control and PRP mice at 7 days (Fig. 2A), 14 days (Fig. 2B) and 28 days (Fig. 2C) indicated that the number of mature osteoclasts in the control group was higher than that in the PRP group. This indicated a significant attenuation of osteoclast activity through treatment with PRP. OcN/BP was determined by TRAP staining to quantify reduction in osteoclasts. Goldner's trichrome staining indicated an obvious reduction in osteoclasts and an increase in osteoblasts quantified as the OcN/BP and ObN/BP (Fig. 2). These results indicated that PRP inhibits osteoclast formation
Table I. Downregulated genes according to the gene chip analysis.

\begin{tabular}{|c|c|c|c|}
\hline Gene & P-value & Probe name & Fold change \\
\hline $\mathrm{Wnt}^{\mathrm{a}}$ & 0.02624592 & A_52_P361534 & 3.4888073 \\
\hline $\mathrm{Dkk}^{\mathrm{a}}$ & 0.03172296 & A_51_P379069 & 9.9720192 \\
\hline Fzd $^{a}$ & 0.04910161 & A_52_P597634 & 2.0385147 \\
\hline $\mathrm{Fzd}^{\mathrm{a}}$ & 0.02590137 & A_51_P404077 & 7.5377999 \\
\hline Dvl2 & 0.00029402 & A_55_P2055869 & 3.1892181 \\
\hline Ctbp2 & 0.01244548 & A_52_P15212 & 3.1172611 \\
\hline Wnt8b $b^{a}$ & 0.00420996 & A_55_P2140913 & 2.0766898 \\
\hline Wnt3a $\mathrm{a}^{\mathrm{a}}$ & 0.02285019 & A_55_P2067649 & 2.9224466 \\
\hline Wnt10a ${ }^{a}$ & 0.03544225 & A_51_P171616 & 3.1686486 \\
\hline Vangl2a & 0.03769394 & A_55_P2117984 & 2.0264866 \\
\hline Csnk2a $2^{\mathrm{a}}$ & 0.03733953 & A_52_P207614 & 5.0116083 \\
\hline Adm & 0.04438596 & A_51_P265571 & 8.1618855 \\
\hline Shh & 0.03644892 & A_52_P49014 & 3.8205879 \\
\hline Ucp1 & $5.9393 \times 10-5$ & A_51_P426353 & 16.0701171 \\
\hline Hnrnpr & 0.01911658 & A_55_P1969625 & 6.9950514 \\
\hline Rest & 0.0265632 & A_55_P2143306 & 6.1341825 \\
\hline Hmga2 & 0.00308199 & A_52_P300730 & 5.2488875 \\
\hline $\mathrm{Cd} 44$ & 0.03947195 & A_55_P2166501 & 5.1884358 \\
\hline Areg & 0.00801028 & A_52_P482897 & 4.3739025 \\
\hline Vcam1 & 0.03508028 & A_52_P520495 & 4.4398901 \\
\hline Sema3a & 0.03332904 & A_55_P2054013 & 4.4616508 \\
\hline
\end{tabular}

${ }^{\mathrm{a}}$ Genes involved in Wnt pathway. Highly downregulated genes, which were reported to be associated with osteoclasts in PRP-treated mice, in comparison with those in the control group are presented based on microarray data. Dkk1 was the focus of the present study. Dkk1, Dickkopf-related protein 1.

and promotes osteoblast formation in the TMJ of mice with mandibular protrusion.

PRP suppresses RANKL-induced osteoclast differentiation but enhances osteogenesis. To further investigate the role of PRP in osteoclast differentiation, BMMs were cultured with M-CSF $(20 \mathrm{ng} / \mathrm{ml})$ and RANKL $(20 \mathrm{ng} / \mathrm{ml})$, in the presence or absence of $1 \%$ PRP. Four days later, cells were fixed and subjected to TRAP staining. As expected, the number of TRAP-positive multinucleated cells per field was significantly lower in the PRP group as compared with that in the control group. Alizarin Red S staining of BMSCs cultured with PRP for 12 days indicated a higher number of mineralization nodules compared with that in the control group (Fig. 3A). These results indicated that PRP inhibited RANKL-induced osteoclast differentiation and accelerated osteoblastic activity, which induced a positive effect on bone regeneration.

PRP inhibits bone resorption by downregulation of osteoclastassociated genes. The expression levels of RANKL-induced differentiation marker genes NFATc1, TRAP and c-fos, as well as those of the resorptive activity marker genes Ctsk, CAR2 and MMP9 were assessed by RT-qPCR in osteoclasts after 

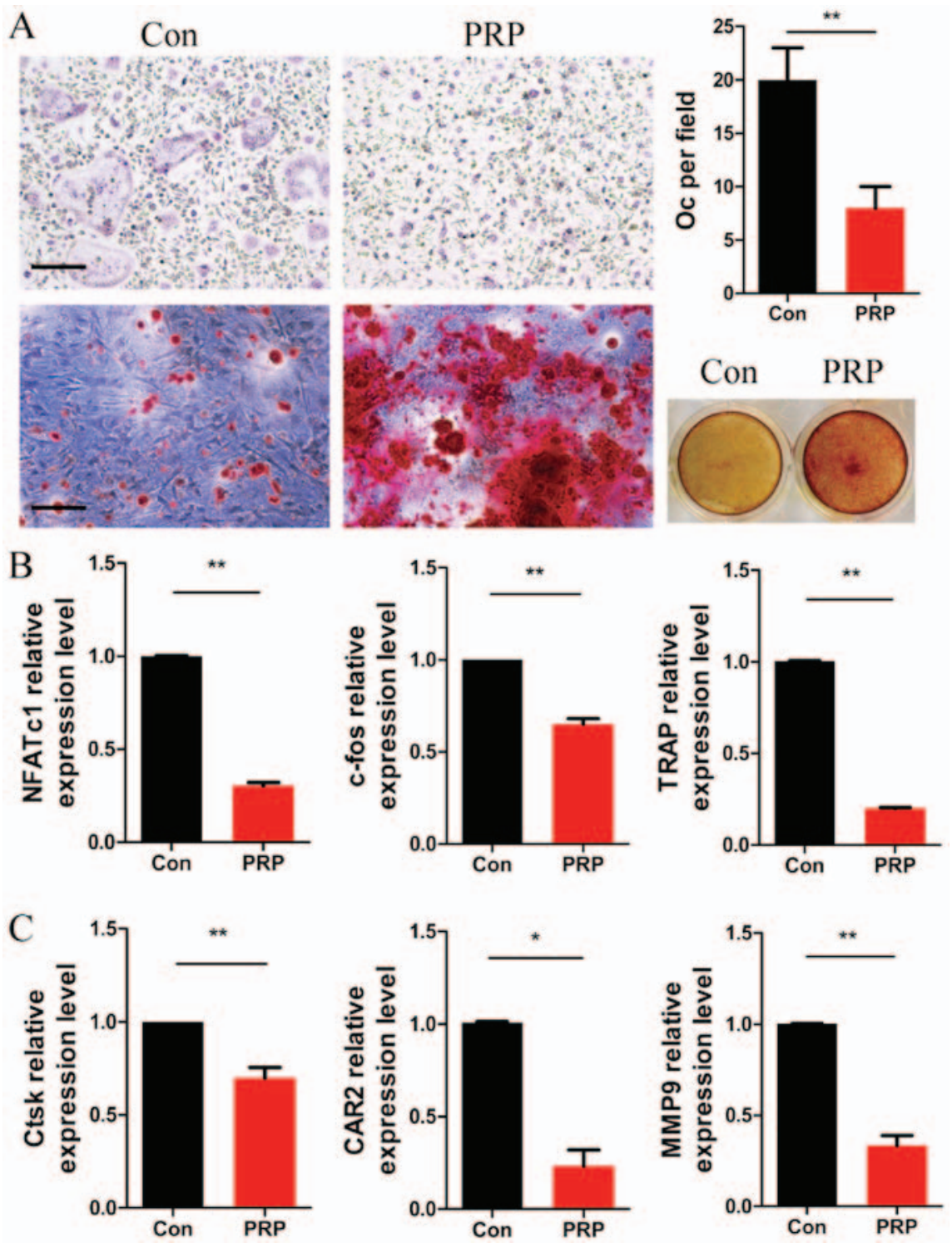

Figure 3. PRP suppresses the early stage of RANKL-induced osteoclastogenesis but enhances osteoblast differentiation. (A) Bone marrow-derived macrophages were cultured with macrophage colony stimulating factor $(20 \mathrm{ng} / \mathrm{ml})$ and RANKL $(20 \mathrm{ng} / \mathrm{ml})$ in the presence or absence of $1 \%$ PRP for 3 days. The cells were fixed, permeabilized and stained for TRAP. Images were captured under a light microscope (top left) and the number of TRAP-positive osteoclasts (>3 nuclei) per field was determined (top right). Scale bar, $200 \mu \mathrm{m}$. Bone marrow stromal cells were stained with Alizarin red and examined under a microscope (magnification, x100; bottom left) and the wells of 12-well plates were observed under image scanner (bottom right). (B and C) Effect of PRP on NFATc1, c-fos, TRAP, Ctsk, CAR2 and MMP9 mRNA expression in osteoclasts. All values are expressed as the mean \pm standard deviation $(\mathrm{n}=3)$. " $\mathrm{P}<0.05$ and ${ }^{* *} \mathrm{P}<0.01$. PRP, platelet-rich plasma; RANKL, receptor activator of nuclear factor- $\mathrm{kB}$ ligand; TRAP, tartrate resistant acid phosphatase; Oc, osteoclasts; NFATc1, nuclear factor of activated T-cells, cytoplasmic 1; Ctsk, cathepsin k; CAR2, carbonic anhydrase 2; MMP9, matrix metalloproteinase 9.

stimulation with PRP for 3 days. At day 3 of PRP treatment, the expression of NFATc1, TRAP and c-fos was significantly lower than that in the control group (Fig. 3B). Furthermore, downregulation of the expression of Ctsk, CAR2 and MMP9 was observed in the PRP group compared with that in the control group (Fig. 3C). These results indicated that PRP inhibited osteoclast differentiation at the gene expression level.

Gene chip analysis. Based on the assumption that PRP may influence gene expression, gene expression profile chip analysis was used to compare the differences in gene expression between the control and PRP groups. Thousands of differentially expressed transcripts were identified, including 18 genes that exhibited a 100 -fold upregulation and 21 genes that had been previously reported to be associated with osteoclasts exhibiting a downregulation of $>2$-fold (Table I). Furthermore, GO analysis provided terms of enriched biological process, cellular component and molecular function of the differentially expressed genes, the top 9 of which are presented in Fig. 4B. These were associated with cell adhesion molecules (CAMs), basal cell carcinoma, spliceosome, the Hedgehog signaling pathway, the Notch signaling pathway, pathways in cancer, tight junction, the Wnt signaling pathway and transcriptional misregulation in cancer. The Wnt signaling pathway, which is considered important for osteoblast differentiation, ranked eighth. The activation of Wnt signaling indicates that osteoblast differentiation is enhanced by PRP. Results of Alizarin Red S staining were also consistent with this hypothesis. The effect of PRP on osteoblasts is 
A

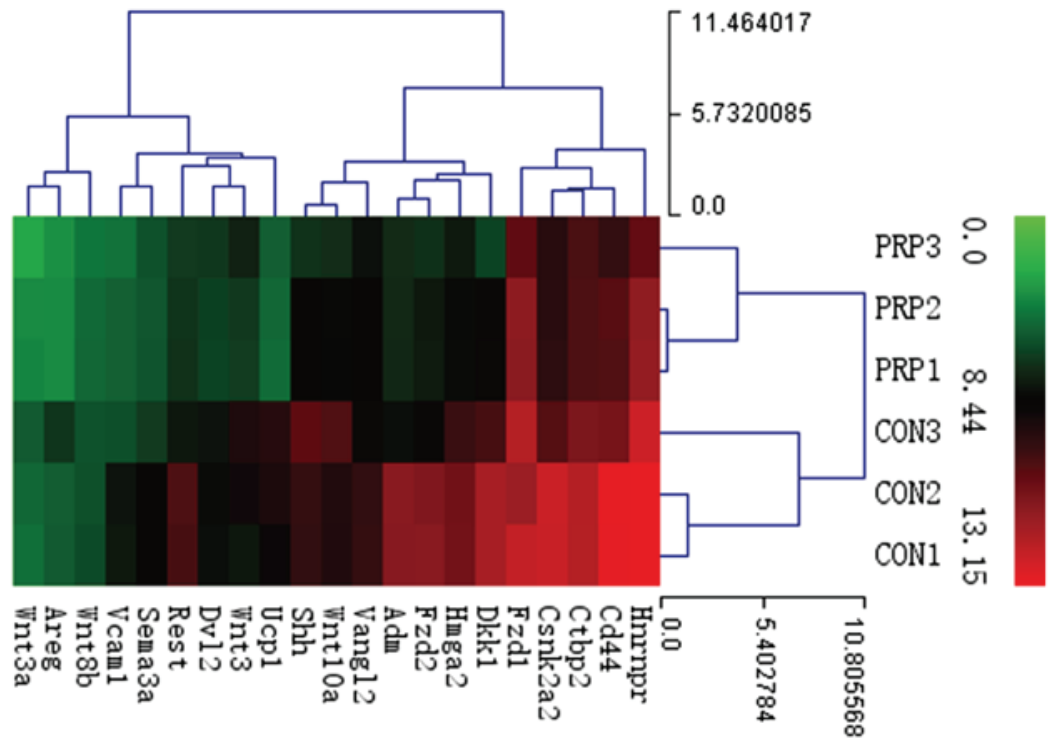

B

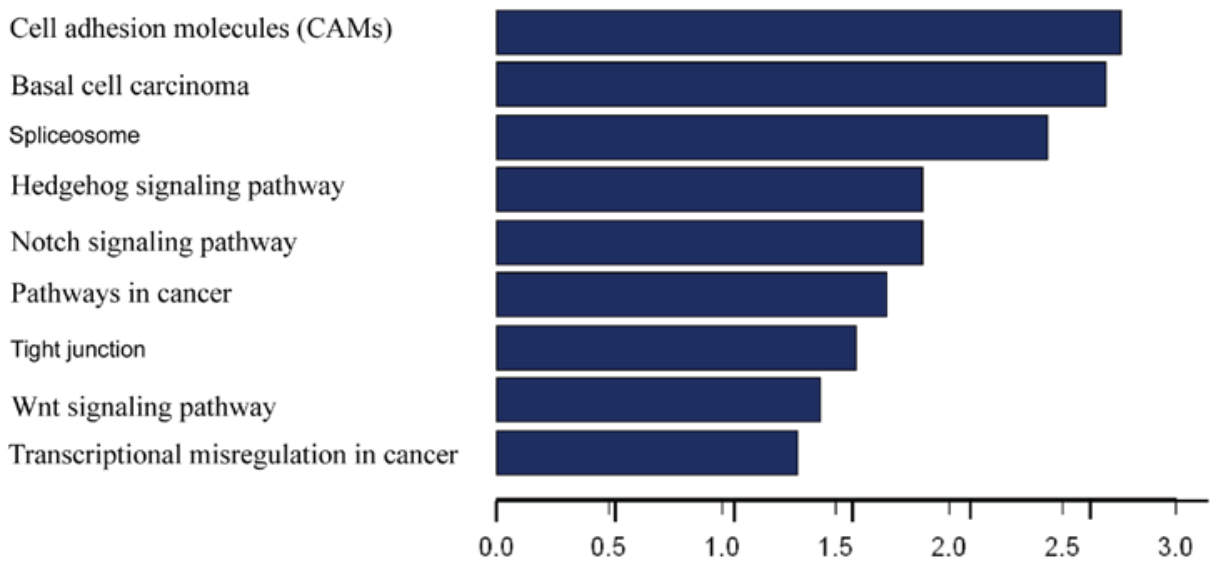

Figure 4. Gene chip analysis. (A) Heat map indicating the hierarchical clustering of differentially expressed genes in the samples treated with PRP in comparison with that in the control groups. Each row represents one gene and each column represents one group. The expression levels are represented by the following colors: Red indicates high relative expression and green signifies low relative expression. (B) Top 9 downregulated pathways based on Kyoto Encyclopedia of Genes and Genomes and Biocarta databases. The enrichment score equals the -Log P-value. PRP, platelet-rich plasma; CAM, cell adhesion molecule; Con, control.

well documented in the literature $(12,24)$. Dkk1 is a known inhibitor of Wnt signaling, which was demonstrated to impair osteoblast activity and potentially stimulate osteoclastogenesis (25). Therefore, among the downregulated genes, the present study focused on the Dkk1 gene, which exhibited a 9.972-fold change in expression. The Wnt pathway was selected for further research.

Wnt signaling pathway is activated during osteoclast differentiation. To further explore the molecular mechanisms of the anti-osteoclastic effects of PRP, RNA and protein from cells pretreated with PRP was assessed for the expression of Wnt-associated signaling molecules by RT-qPCR and western blot analysis, respectively. Of note, $\beta$-catenin (a component of the canonical Wnt pathway) is known to have a critical role in bone formation and remodeling (26). Cyclin D1, the downstream transcription factor of $\beta$-catenin, has a mediatory role in the canonical Wnt pathway. Dkk1 is a specific inhibitor of the canonical Wnt pathway. PRP was demonstrated to significantly reduce RANKL-induced osteoclast differentiation by regulating early signaling pathways and increasing the levels of $\beta$-catenin and cyclin D1 (27). Downregulation of Dkk1 and upregulation of $\beta$-catenin indicated that PRP inhibited osteoclast differentiation by activating the $\beta$-catenin/Wnt pathway (Fig. 5). These results indicated that PRP causes a repression of Dkk1, which induces the Wnt signaling pathway. This results in inhibition of phosphorylation of $\beta$-catenin, leading to the accumulation of non-phosphorylated $\beta$-catenin in the cytoplasm and its translocation to the nucleus. This in turn induces upregulation of the downstream target gene cyclin D1 (Fig. 6).

\section{Discussion}

Various studies have demonstrated condylar cartilage remodeling and increases in mandibular length after insertion of functional appliances at an early stage of growth and development $(28,29)$. In treatment of class II occlusion, functional appliances produce an impact on mandibular advancement, which in turn alters the biophysical environment in the TMJ and induces a series of cellular changes (30). The increase in new bone formation in the TMJ as a result of mandibular 
A
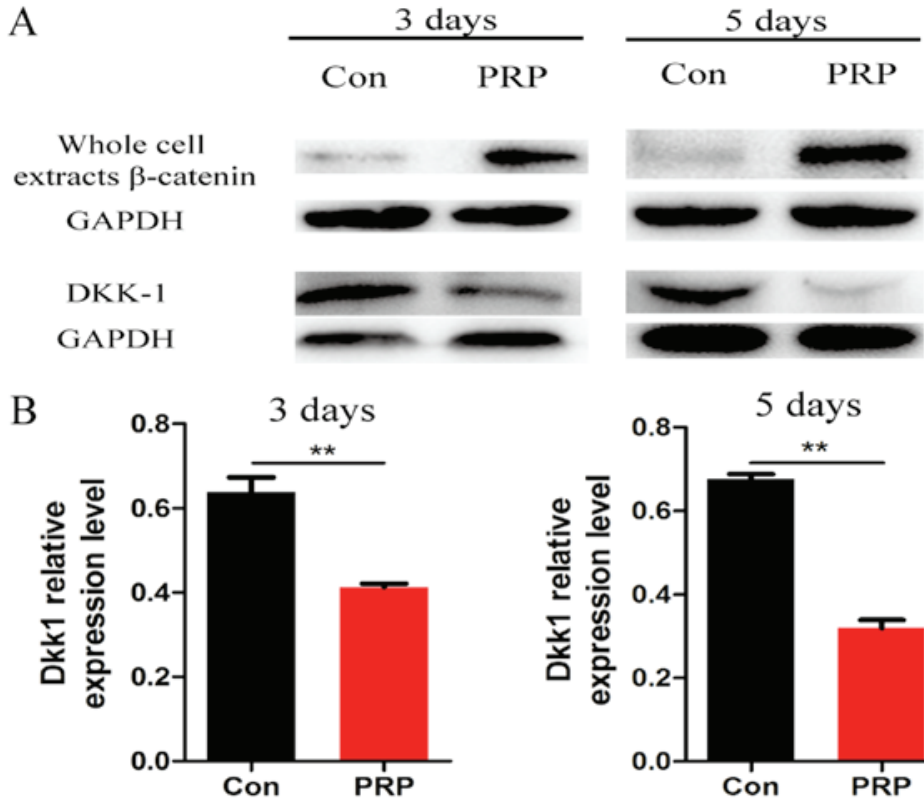

C
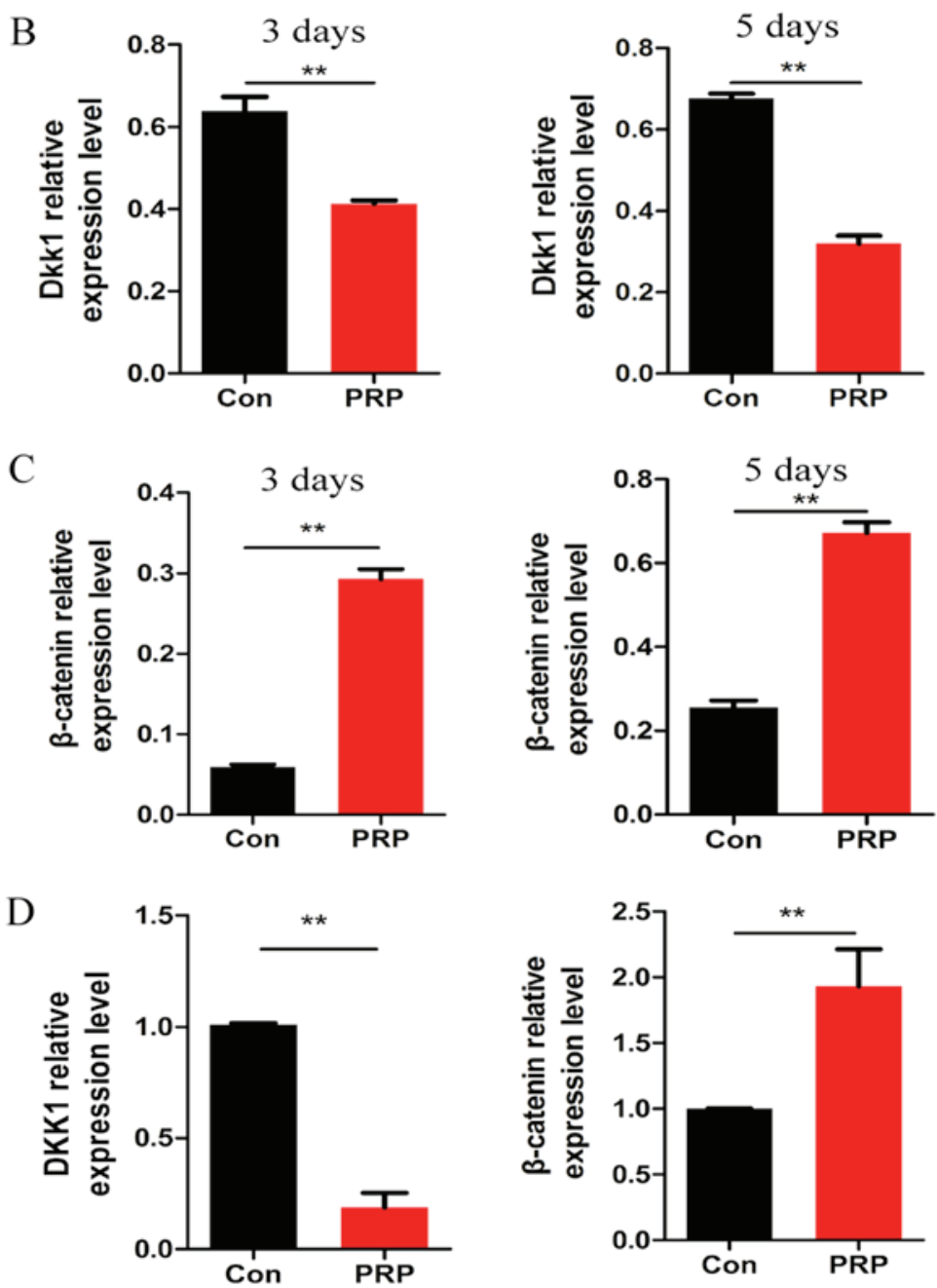

Figure 5. The Wnt signaling pathway is activated during osteoclast differentiation. PRP increased the induction of $\beta$-catenin and reduced Dkk1 in RANKL-treated osteoclasts. Bone marrow-derived macrophages were incubated with macrophage colony stimulating factor ( $20 \mathrm{ng} / \mathrm{ml})$, RANKL (20 ng/ml) and $1 \%$ PRP for the indicated durations. (A) The protein expression levels of Dkk1 and $\beta$-catenin were determined by western blot analysis. (B and C) Protein bands of Dkk1 and $\beta$-catenin were quantified by densitometry, revealing that the expression of Dkk1 was declined while $\beta$-catenin was increased compared with that in the control group at days 3 and 5 ( $\mathrm{P}<0.01)$. (D) PRP treatment for 3 days increased $\beta$-catenin mRNA expression, while it decreased Dkk1 mRNA expression in the PRP-treated group during osteoclast differentiation, which was well consistent with the results of the western blot analysis. Values are expressed as the mean \pm standard deviation $(\mathrm{n}=3) .{ }^{* *} \mathrm{P}<0.01$. PRP, platelet-rich plasma; RANKL, receptor activator of nuclear factor- $\mathrm{kB}$ ligand; Dkk1, Dickkopf-related protein 1; Con, control.

advancement has been reported to cause adaptive remodeling of the mandibular condyle. TMJ reconstruction relies on the fine balance between ossification and bone resorption.

PRP was first used to enhance bone grafts for the repair of mandibular defects (31). Ever since, PRP has found wide applications in medicine for bone tissue engineering, regeneration of damaged joints, oral maxillofacial surgery and oral planting due to its self-replicating ability, multi-directional differentiation potential, easy availability, absence of immune rejection and minimal ethical concerns (32).
In the present study, PRP was identified as a critical negative regulator of osteoclastogenesis and bone resorption in vivo and in vitro. First, micro-CT and histological techniques were used to observe the morphology of the TMJs of the experimental mice. The outcomes of micro-CT and histological analyses for the two groups revealed significant differences in parameters including bone mass, $\mathrm{OcN}$ and $\mathrm{ObN}$. It was thus hypothesized that PRP may affect bone formation by inhibiting osteoclast differentiation. To confirm this hypothesis, RT-qPCR was performed to assess the expression of osteoclast 


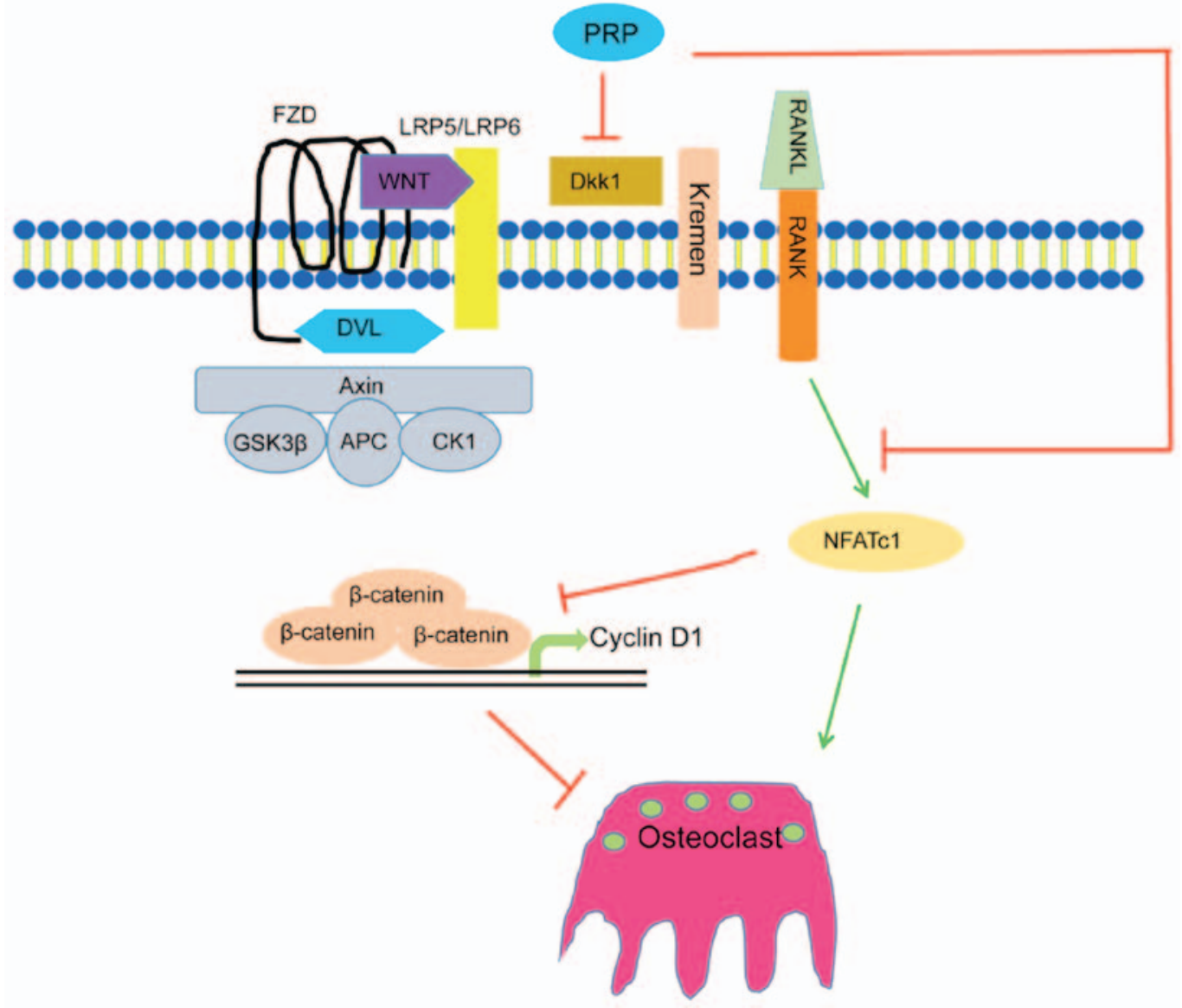

Figure 6. Schematic illustration of the WNT signaling pathway activated by PRP. PRP inhibited the expression of Dkk1, which accelerates Wnt ligand binding with the Wnt receptor. PRP upregulated the expression of NFATc1, which suppresses phosphorylation of $\beta$-catenin. Cyclin D1 is one of the target genes of $\beta$-catenin, and $\beta$-catenin and NFATc1 contribute to osteoclast differentiation by combination of RANKL and RANK. PRP, platelet-rich plasma; RANKL, receptor activator of nuclear factor-kB ligand; Dkk1, dickkopf-related protein 1; NFATc1, nuclear factor of activated T-cells, cytoplasmic 1.

marker genes (NFATc1, c-fos, TRAP, Ctsk, CAR2 and MMP9). Downregulation of c-fos and NFATc1 mRNA is indicative of the inhibition of osteoclast differentiation (33). As expected, PRP repressed the expression of RANKL-induced differentiation marker genes. In addition, gene chip analysis identified several differentially expressed genes, among which Dkk1 was of particular interest.

The results of the present study provide insight into the link between PRP and osteoclast differentiation. The Wnt signaling pathway was identified to be involved in PRP-induced inhibition of osteoclast differentiation. To further validate this result, RANKL-induced osteoclasts treated with PRP were examined for the expression of Dkk1 and $\beta$-catenin using RT-qPCR and western blot analysis. The results demonstrated that PRP affected Dkk1 and $\beta$-catenin expression during osteoclast differentiation, which confirmed the involvement of the Wnt pathway in PRP-mediated inhibition of osteoclast differentiation. Several studies have investigated the role of PRP in inducing bone formation via promotion of osteoblastic differentiation (34). Several lignin-like compounds have been reported to repress RANKL-induced osteoclastogenesis and inhibit osteoclast-mediated bone resorption activity (35). The present study established extensive functions of PRP in mouse TMJ remodeling, and it was indicated that local injection of PRP in the damaged domain may be effective in inhibiting TMJ remodeling, or may at least provide symptom relief.
There are several limitations to the present study. Firstly, no sham group was included for comparison. Secondly, histologic results in the control and PRP groups were not time-dependent (days 7, 14 and 28), which indicates that there may be a complicated mechanism at work, which requires further research.

PRP has important effects not only on osteoblastic differentiation but also on osteoclast differentiation during bone formation. However, the present study did not examine the interaction between osteoblasts and osteoclasts in bone formation. Further study is therefore required to identify additional details regarding this potential association. To date, no uniform methods and standards for the extraction of PRP have been established, which makes it difficult to obtain and apply it at production level quantities (36). Further research will be performed to identify the precise elements involved in the influence of PRP on bone formation.

\section{Acknowledgements}

This study was supported by the National Natural Science Foundation of China (grant no. 81371179), the Natural Science Foundation of Jiangsu Province (grant no. BK20150048) and a Project Funded by the Priority Academic Program Development of Jiangsu Higher Education Institutions (grant no. 2014-037). 


\section{References}

1. Cozza P, Baccetti T, Franchi L, De Toffol L and McNamara JA Jr: Mandibular changes produced by functional appliances in Class II malocclusion: A systematic review. Am J Orthod Dentofacial Orthop 129: 599.e1-599.e12, 2006.

2. Owtad P, Potres Z, Shen G, Petocz P and Darendeliler MA: A histochemical study on condylar cartilage and glenoid fossa during mandibular advancement. Angle Orthod 81: 270-276, 2011 .

3. Leung FY, Rabie AB and Hägg U: Neovascularization and bone formation in the condyle during stepwise mandibular advancement. Eur J Orthod 26: 137-141, 2004.

4. Feres MF, Alhadlaq A and El-Bialy T: Adjunctive techniques for enhancing mandibular growth in class II malocclusion. Med Hypotheses 84: 301-304, 2015.

5. Ruf S and Pancherz H: Temporomandibular joint remodeling in adolescents and young adults during Herbst treatment: A prospective longitudinal magnetic resonance imaging and cephalometric radiographic investigation. Am J Orthod Dentofacial Orthop 115: 607-618, 1999

6. Rho J, Takami M and Choi Y: Osteoimmunology: Interactions of the immune and skeletal systems. Mol Cells 17: 1-9, 2004.

7. Boyle WJ, Simonet WS and Lacey DL: Osteoclast differentiation and activation. Nature 423: 337-342, 2003.

8. Teitelbaum SL and Ross FP: Genetic regulation of osteoclast development and function. Nat Rev Genet 4: 638-649, 2003.

9. Yasuda H, Shima N, Nakagawa N, Yamaguchi K, Kinosaki M, Mochizuki S, Tomoyasu A, Yano K, Goto M, Murakami A, et al: Osteoclast differentiation factor is a ligand for osteoprotegerin/osteoclastogenesis-inhibitory factor and is identical to TRANCE/RANKL. Proc Natl Acad Sci USA 95: 3597-3602, 1998

10. Foster TE, Puskas BL, Mandelbaum BR, Gerhardt MB and Rodeo SA: Platelet-rich plasma: From basic science to clinical applications. Am J Sports Med 37: 2259-2272, 2009.

11. Tomoyasu A, Higashio K, Kanomata K, Goto M, Kodaira K, Serizawa H, Suda T, Nakamura A, Nojima J, Fukuda T, et al: Platelet-rich plasma stimulates osteoblastic differentiation in the presence of BMPs. Biochem Biophys Res Commun 361: 62-67, 2007.

12. Kanno T, Takahashi T, Tsujisawa T, Ariyoshi $\mathrm{W}$ and Nishihara $\mathrm{T}$ : Platelet-rich plasma enhances human osteoblast-like cell proliferation and differentiation. J Oral Maxillofac Surg 63: 362-369, 2005.

13. Tagliaro ML, Rassi Guimarães ML, Pereira Padilha DM, Callegari-Jacques SM and Jeckel-Neto EA: Mandibular advancement and morphological changes in the mandibles of female mice of different ages. Exp Gerontol 41: 1157-1164, 2006.

14. Ba R, Wei J, Li M, Cheng X, Zhao Y and Wu W: Cell-bricks based injectable niche guided persistent ectopic chondrogenesis of bone marrow-derived mesenchymal stem cells and enabled nasal augmentation. Stem Cell Res Ther 6: 16, 2015.

15. Rafferty KL, Liu ZJ, Ye W, Navarrete AL, Nguyen TT, Salamati A and Herring SW: Botulinum toxin in masticatory muscles: Short-and long-term effects on muscle, bone, and craniofacial function in adult rabbits. Bone 50: 651-662, 2012.

16. Ma J, Chen W, Zhang L, Tucker B, Zhu G, Sasaki H, Hao L, Wang L, $\mathrm{Ci} \mathrm{H}$, Jiang $\mathrm{H}$, et al: RNA interference-mediated silencing of Atp6i prevents both periapical bone erosion and inflammation in the mouse model of endodontic disease. Infect Immun 81: 1021-1030, 2013.

17. Chen W, Ma J, Zhu G, Jules J, Wu M, McConnell M, Tian F, Paulson $\mathrm{C}$, Zhou $\mathrm{X}$, Wang L, et al: $\mathrm{Cbf} \beta$ deletion in mice recapitulates cleidocranial dysplasia and reveals multiple functions of $\mathrm{Cbf} \beta$ required for skeletal development. Proc Natl Acad Sci USA 111: 8482-8487, 2014

18. Bendre MS, Gaddy-Kurten D, Mon-Foote T, Akel NS, Skinner RA, Nicholas RW and Suva LJ: Expression of interleukin 8 and not parathyroid hormone-related protein by human breast cancer cells correlates with bone metastasis in vivo. Cancer Res 62: 5571-5579, 2002.
19. Yamachika E, Tsujigiwa H, Matsubara M, Hirata Y, Kita K, Takabatake K, Mizukawa N, Kaneda Y, Nagatsuka H and Iida S: Basic fibroblast growth factor supports expansion of mouse compact bone-derived mesenchymal stem cells (MSCs) and regeneration of bone from MSC in vivo. J Mol Histol 43: 223-233, 2012.

20. Lee YD, Yoon SH, Park CK, Lee J, Lee ZH and Kim HH: Caveolin-1 regulates osteoclastogenesis and bone metabolism in a sex-dependent manner. J Biol Chem 290: 6522-6530, 2015.

21. Edgar R, Domrachev M and Lash AE: Gene Expression Omnibus: NCBI gene expression and hybridization array data repository. Nucleic Acids Res 30: 207-210, 2002.

22. Livak KJ and Schmittgen TD: Analysis of relative gene expression data using real-time quantitative PCR and the 2(-Delta Delta C(T)) method. Methods 25: 402-408, 2001.

23. Lee HY, Lee SY, Kim SD, Shim JW, Kim HJ, Jung YS, Kwon JY, Baek SH, Chung J and Bae YS: Sphingosylphosphorylcholine stimulates CCL2 production from human umbilical vein endothelial cells. J Immunol 186: 4347-4353, 2011.

24. Li H, Sun S, Liu H, Chen H, Rong X, Lou J, Yang Y, Yang Y and Liu H: Use of a biological reactor and platelet-rich plasma for the construction of tissue-engineered bone to repair articular cartilage defects. Exp Ther Med 12: 711-719, 2016

25. Rachner TD, Göbel A, Benad-Mehner P, Hofbauer LC and Rauner M: Dickkopf-1 as a mediator and novel target in malignant bone disease. Cancer Lett 346: 172-177, 2014

26. Willert K and Nusse R: Beta-catenin: A key mediator of Wnt signaling. Curr Opin Genet Dev 8: 95-102, 1998

27. Wei W, Zeve D, Suh JM, Wang X, Du Y,Zerwekh JE, Dechow PC, Graff JM and Wan Y: Biphasic and dosage-dependent regulation of osteoclastogenesis by $\beta$-catenin. Mol Cell Biol 31: 4706-4719, 2011.

28. Saikoski LZ, Cançado RH, Valarelli FP and de Freitas KM: Dentoskeletal effects of Class II malocclusion treatment with the Twin Block appliance in a Brazilian sample: A prospective study. Dental Press J Orthod 19: 36-45, 2014.

29. Baysal A and Uysal T: Dentoskeletal effects of Twin Block and Herbst appliances in patients with Class II division 1 mandibular retrognathy. Eur J Orthod 36: 164-172, 2014.

30. Rabie AB, Zhao Z, Shen G, Hägg EU, Dr O and Robinson W: Osteogenesis in the glenoid fossa in response to mandibular advancement. Am J Orthod Dentofacial Orthop 119: 390-400, 2001.

31. Marx RE, Carlson ER, Eichstaedt RM, Schimmele SR, Strauss JE and Georgeff KR: Platelet-rich plasma: Growth factor enhancement for bone grafts. Oral Surg Oral Med Oral Pathol Oral Radiol Endod 85: 638-646, 1998.

32. Nathani DB, Sequeira J and Rao BH: Comparison of platelet rich plasma and synthetic graft material for bone regeneration after third molar extraction. Ann Maxillofac Surg 5: 213-218, 2015.

33. Xu X, Liu N, Wang Y, Pan LC, Wu D, Peng Q, Zhang M, Wang HB and Sun WC: Tatarinan O, a lignin-like compound from the roots of Acorus tatarinowii Schott inhibits osteoclast differentiation through suppressing the expression of c-Fos and NFATc1. Int Immunopharmacol 34: 212-219, 2016.

34. Goto H, Matsuyama T, Miyamoto M, Yonamine Y and Izumi Y: Platelet-rich plasma/osteoblasts complex induces bone formation via osteoblastic differentiation following subcutaneous transplantation. J Periodontal Res 41: 455-462, 2006.

35. Ito S, Ohmi A, Sakamiya A, Yano T, Okumura K, Nishimura N and Kagontani K: Ginger hexane extract suppresses RANKLinduced osteoclast differentiation. Biosci Biotechnol Biochem 80: 779-785, 2016.

36. Anitua E, Sánchez M, Orive G and Andía I: The potential impact of the preparation rich in growth factors (PRGF) in different medical fields. Biomaterials 28: 4551-4560, 2007.

This work is licensed under a Creative Commons Attribution-NonCommercial-NoDerivatives 4.0 International (CC BY-NC-ND 4.0) License. 